\title{
CNN-SICE Learner Based Image Contrast Enhancement
}

\author{
Pooja Patel \\ Department of CSE, Sagar Institute of Research \& Technology (SIRT), Bhopal, India
}

Corresponding author: drsourabhverma12@gmail.com

\begin{abstract}
Producing the natural scene with good contrast, vivid color and rich details is an essential goal of digital photography. The acquired images, however, are often under-exposed or over-exposed because of poor lighting conditions and the limited dynamic range of imaging device. Contrast enhancement is thus an important step to improve the quality of recorded images and make the image details more visible. Many research work have been done for image enhancement. In this paper, different techniques and algorithms using machine learning approach are studied and Block based CNN Learner is designed for contrast enhancement.
\end{abstract}

Keywords: Image enhancement, Image quality, Machine learning approaches, Digital image processing.

Image processing is an essential and promising research area in various real time application fields such as medical imaging, video surveillance, industrial X ray imaging, oceanography etc..$^{[1]}$. Image enhancement is a preprocessing technique in many image processing applications that can produce an improved quality image than the original image so that the output image is more suitable for analysis by human or machine in specific applications. In general, image enhancement techniques are divided into two broad categories, such as spatial techniques and frequency domains. In spatial domain techniques, the pixels themselves are modified directly to improve an image. In frequency domain method, modification is done on the Fourier transformed image and inverse Fourier transform is applied to the modified image to get the enhanced image. Quality of the image gets affected by uneven or poor illumination, external atmospheric condition such as fog or haze, wrong lens aperture setting of the camera, noise etc.

So, these degraded quality images are improved by increasing the brightness and contrast, by de-noising the image through various enhancement techniques. Researchers have developed numerous enhancement techniques that are good in enhancing the contrast of an image, while some are good for de-noising the images. In real time applications an enhancement technique should be capable of enhancing real color images in lesser time with lesser computational cost by reducing (i) the effect of haze or fog, (ii) poor or 
uneven illumination effect on an image and (iii) noise introduced in an image. This research review work focus on various color image enhancement techniques that improves the contrast of real time images. Requirements of real time image enhancement techniques ${ }^{[2]}$ are; (i) It should be adaptive in nature (i.e.) should be able to enhance any type of images for a specific application, (ii) Should enhance a image in less processing time, (iii) It should utilize less computational resources.

Image enhancement techniques have been widely used in many applications of image processing. Contrast is an important factor in any subjective evaluation of image quality. Contrast is created by the difference in luminance reflected from two adjacent surfaces. In other words, contrast is the difference in visual properties that makes an object distinguishable from other objects and the background.

In visual perception, contrast is determined by the difference in the color and brightness of the object with other objects. If the contrast of an image is highly concentrated on a specific range, e.g. an image is very dark. The information may be lost in those areas which are excessively and uniformly concentrated. The problem is to optimize the contrast of an image in order to represent all the information in the input image.

Low contrast: Image values concentrated near a narrow range (mostly dark, or mostly bright, or mostly medium values).

Contrast enhancement: Change the image value change the image value distribution to cover a wide range Contrast of an image can be revealed by its histogram.

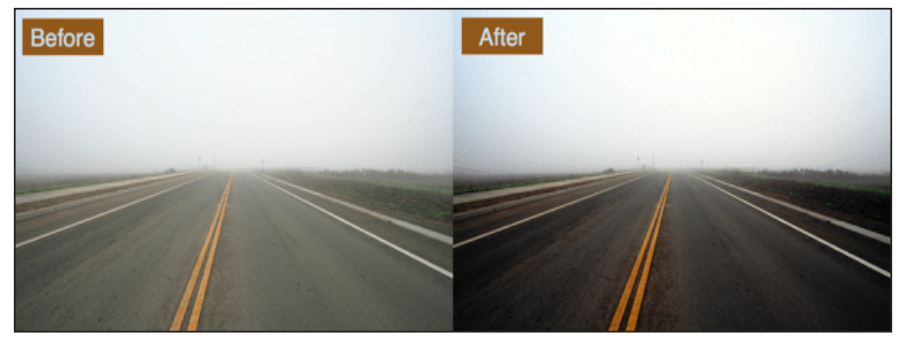

Fig. 1: Contrast Enhancement of Image

Histogram of a monochrome image with L possible gray levels,

$f=0,1, \ldots, L-1$.

$=P(1)=n_{l} / n$

Where,

$n_{1}=$ Number of pixels with gray level $1 . n=$ Total number of pixels in the image

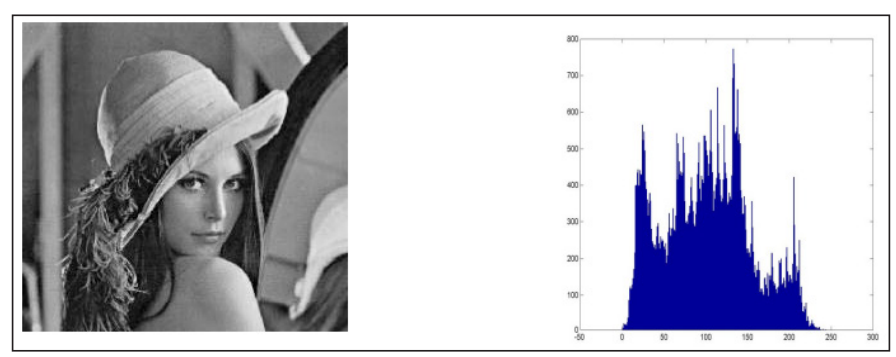

Fig. 2: Histogram of an Image 


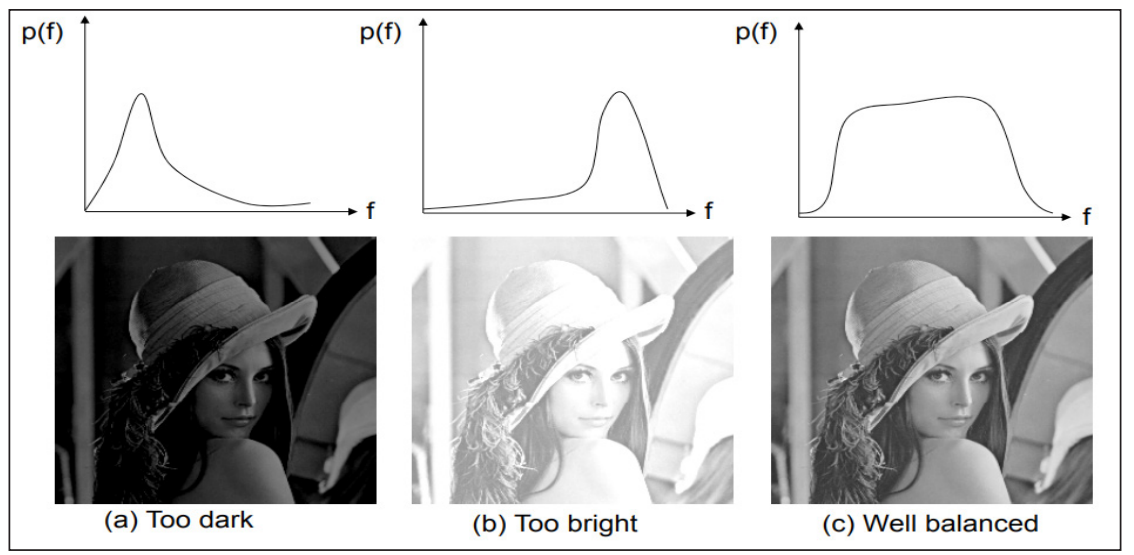

Fig. 3: Examples of Histograms

\section{Related Work}

Image enhancement is a process by which we can improve the quality of the digital image which makes it easier for identifying features. This can be done by removing noise, sharpening, or brightening an image. The techniques identified in the paper to enhance an image are contrast stretching and image sharpening, nonlinear image enhancement technique, genetic algorithm, generalized fuzzy enhancement, wavelet transform technique, multi-scale and single-scale retinex improvement technique, etc. ${ }^{[3]}$.

$\mathrm{In}^{[4]}$, Negi et al., discussed contrast stretching and image sharpening techniques. It is an approach that concurrently adjusts contrast and enhances boundaries of an image. On the gray-scale image contrast stretching is applied and then it proceeds to Laplacian mask, and finally, Laplacian image is appended to the original gray-scale image to obtain the desired sharpened image.

$\mathrm{In}^{[5]}$, Wu et al., proposed image enhancement using wavelet-based contourlet transform with cycle translation. In this, wavelet transform is used for decreasing the redundancy occurring in the original method of contourlet transform. WBCT and cycle translation are merged. At last, to magnify the images, adaptive enhancement function is selected. The proposed method can efficiently magnify the images and decrease the flecked at the background region, the image edges.

$\mathrm{In}^{[6]}$ Gupta et al., discussed image enhancement and segmentation techniques. The paper concluded that segmentation is considered as one of the main steps in image processing. It divides a digital image into multiple regions in order to analyze them. It is also used to distinguish different objects in the image.

$\mathrm{In}^{[7]}$, Wang et al., discussed NIE (Nonlinear Image Enhancement). Simulation and identification processes are used along with the proposed NIE method. This process uses clipping and scaling parameters which are an appropriate combination of various images. This process enhances the quality of blurred image and a better quality is achieved, and PSNR (signal-to-noise ratio) performance is obtained than other nonlinear enhancement techniques.

$\mathrm{In}^{[8]}$, Premkumar et al., discussed the color image contrast enhancement technique. Firstly, RGB image is transformed to HSV (hue, saturation, and value) color space. Hue color channel is selected for DST decomposition. The lower directional sub band is used for reformation. By transforming HSV to RGB 
color space, the enhanced image is acquired. From proposed DST-based contrast image enhancement approach, the satisfactory result is attained.

$\mathrm{In}^{[9]}$, Shanmugavadivu et al., discussed a contrast enhancement technique using the basic concept of histogram equalization. In this proposed technique, the image histogram is first divided into two parts using the Otsu threshold. Thus, a series of optimized weighing conditions formulated using particle optimization (PSO) is applied to both parts. The two parts are then independently balanced and then combined to produce an optimized contrast image that preserves brightness.

\section{Weaknesses of existing image enhancement techniques}

$\square$ Existing image enhancement algorithms are very computation intensive and require a large amount of memory to store the intermediate data.

$\square$ Algorithms are quite complex to understand and implement.

Very few techniques are practically used for image enhancement which leaves a large scope for new enhancement techniques.

$\square$ Due to the poor lighting condition and limited dynamic range of digital imaging devices, the recorded images are often under/over-exposed and with low contrast.

$\square$ Most of previous single image contrast enhancement (SICE) methods adjust the tone curve to correct the contrast of an input image.

$\square$ Those methods, however, often fail in revealing image details because of the limited information in a single image. On the other hand, the SICE task can be better accomplished if we can learn extra information from appropriately collected training data.

\section{METHODOLOGY}

Single image contrast enhancement (SICE) aims to enhance the visibility of the scene in a very given single low-contrast image. It provides the way to boost the low contrast pictures captured from a high dynamic range scene. several histogram and Retinex based SICE ways are projected within the past decades. Histogram-based ways ${ }^{[4],[5]}$ are wide used due to their simplicity in enhancing low contrast pictures. Those ways plan to distribute the bright intensity on bar chart in a very international or native manner. However, such easy redistribution operations could turn out serious unrealistic effects within the enhanced pictures since they ignore image structural information.

Recently, ways ${ }^{[10]}$ are projected to train a CNN network to map the low dynamic range (LDR) pictures to HDR pictures. $\mathrm{In}^{[10]}$, a $\mathrm{CNN}$ is trained to line the parameters of SICE, that are then used to enhance an input image to a desired image.

With the constructed dataset, the proposed work will designed a block based CNN-SICE enhancer to learn a mapping function between the low contrast input image $\mathrm{I}(\mathrm{x}, \mathrm{y})$ and its corresponding reference image Iref $(\mathrm{x}, \mathrm{y})$. Further, the work train a deep CNN H(I,W). The network is trained with Structural dissimilarity (DSSIM) loss. The DSSIM loss function can be formulated as: 
$\operatorname{DSSIM}(W)=\frac{1}{n} \sum_{i}^{n}\left(1-\operatorname{ssim}\left(I_{\text {ref }}^{(i)}-H\left(I^{(i)}, W\right)\right)\right) / 2$

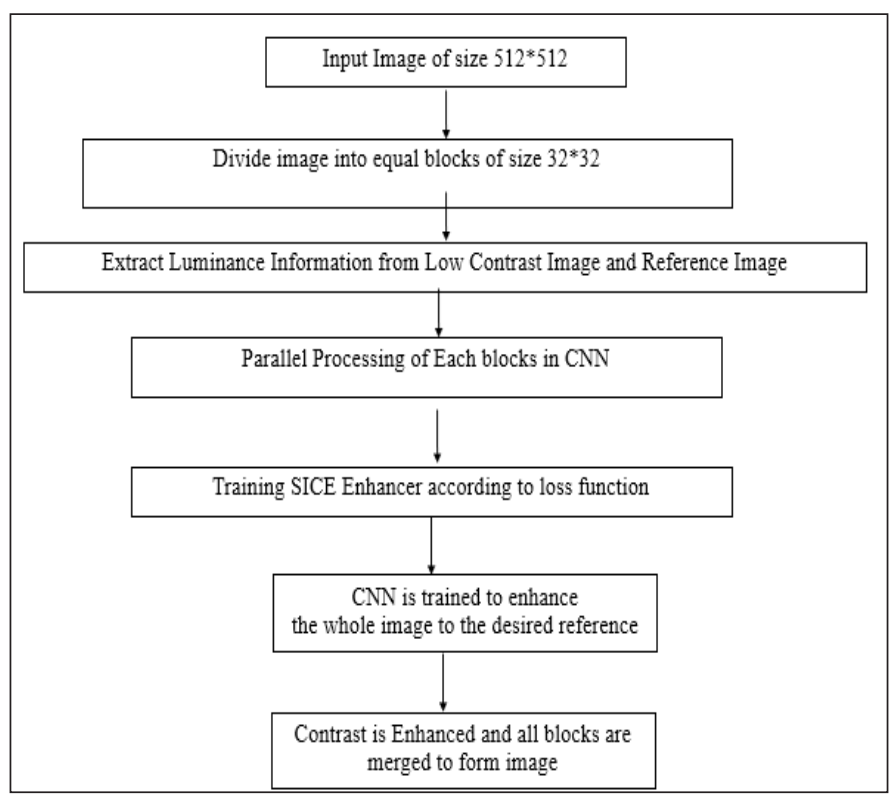

Fig. 4: Proposed Methodology

\section{CONCLUSION}

Image enhancement is an essential preprocessing step in many real time image processing applications. Enhancement of Images is done by many approaches and choice of every approach depends on the type of images. Among all histogram equalization techniques multi histogram equalization techniques improves the contrast and brightness of the images. As analyzed, there are various factors which can affect image quality. Some of them are noise, sharpness, distortion, contrast, color accuracy, dynamic range, exposure accuracy, lens flare, etc. These factors must be kept in mind while choosing or designing any image enhancement algorithm. The proposed CNN-based SICE enhancer, which is capable of adaptively generating high quality enhancement result for a single over-exposed or underexposed input image and may significantly outperforms better to existing work. On processing block parallelly, time complexity is reduced. This work will also be enhanced on videos.

\section{REFERENCES}

1. Singh, P.K., Sangwan, O.P. and Sharma, A. 2013. A Systematic Review on Fault Based Mutation Testing Techniques and Tools for Aspect-J Programs, published in 3rd IEEE International Advance Computing Conference, IACC-2013 at AKGEC Ghaziabad, IEEE Xplore, pp. 1455-1461.

2. Singh, P.K., Panda, R.K. and Sangwan, O.P. 2015. A Critical Analysis on Software Fault Prediction Techniques, published in World Applied Sciences Journal, 33(3): 371-379. 
3. Singh, P. K., Agarwal, D. and Gupta, A.2015. A Systematic Review on Software Defect Prediction, published in Computing for Sustainable Global Development (INDIACom), IEEE, pp. 1793- 97.

4. Negi, S.S. and Bhandari, Y.S.2014. A hybrid approach to Image Enhancement using Contrast Stretching on Image Sharpening and the analysis of various cases arising using histogram, published in Recent Advances and Innovations in Engineering (ICRAIE), pp. 1-6.

5. Wu, C., Liu, Z. and Jiang, H. 2014. Catenary image enhancement using wavelet-based contourlet transform with cycle translation, published in Optik. International Journal for Light and Electron Optics, 125(15): 3922-3925.

6. Tripathi, R. and Gupta, N. 2018. A Review on Segmentation Techniques in Large-Scale Remote Sensing Images. International Journal Online of Science, 4(4), 2018. Retrieved from http://ijoscience.com/ ojsscience/index.php/ojsscience/article/view/143 . Date accessed: 18 December 2018.

7. Wang, L.J. and Huang, Y.C. 2010. Non-linear image enhancement using opportunity costs, published in Second International Conference on Computational Intelligence Communication Systems and Networks (CICSyN), IEEE, pp. 256-261.

8. Premkumar, S. and Parthasarathi, K.A.2014. An efficient approach for colour image enhancement using Discrete Shearlet Transform, published in $2^{\text {nd }}$ International Conference on Current Trends in Engineering and Technology (ICCTET), IEEE, pp. 363-366.

9. Shanmugavadivu, P. and Balasubramanian, K. 2014. Particle swarm optimized multi-objective histogram equalization for image enhancement, published in Optics Laser Technology, 57: 243-251.

10. Jianrui Cai, Shuhang Gu, and Lei Zhang. 2018. "Learning a Deep Single Image Contrast Enhancer from Multi-Exposure Images”, IEEE Transactions on Image Processing, 27(4). 